Mon. Not. R. Astron. Soc. 000, 1-?? (2002) Printed 31 October $2018 \quad$ (MN LATEX style file v2.2)

\title{
Jet-disc coupling through a common energy reservoir in the black hole XTE J1118+480
}

\author{
Julien Malzac ${ }^{1,2 \star}$, Andrea Merloni ${ }^{3}$ and Andrew C. Fabian ${ }^{1}$ \\ ${ }^{1}$ Institute of Astronomy, Madingley Road, Cambridge, CBз OHA \\ ${ }^{2}$ Centre d'Etude Spatiale des Rayonnements, CNRS-UPS, 9 Avenue du Colonel Roche, 31028 Toulouse Cedex 4, France \\ ${ }^{3}$ Max-Planck-Institut für Astrophysik, Karl-Schwarzschild-Strasse 1, D-85741 Garching, Germany
}

Accepted 1988 December 15. Received 1988 December 14; in original form 1988 October 11

\begin{abstract}
We interpret the rapid correlated UV/optical/ X-ray variability of XTE J1118+480 as a signature of the coupling between the X-ray corona and a jet emitting synchrotron radiation in the optical band. We propose a scenario in which the jet and the X-ray corona are fed by the same energy reservoir where large amounts of accretion power are stored before being channelled into either the jet or the high energy radiation. This time dependent model reproduces the main features of the rapid multi-wavelength variability of XTE J1118+480. Assuming that the energy is stored in the form of magnetic field, we find that the required values of the model parameters are compatible with both a patchy corona atop a cold accretion disc and a hot thick inner disc geometry. The range of variability timescales for the X-ray emitting plasma are consistent with the dynamical times of an accretion flow between 10 and 100 Schwarzschild radii. On the other hand, the derived range of timescales associated with the dissipation in the jet extends to timescales more than 10 times larger, confirming the suggestion that the generation of a powerful outflow requires large scale coherent poloidal field structures. A strong requirement of the model is that the total jet power should be at least a few times larger than the observed X-ray luminosity, implying a radiative efficiency for the jet $\epsilon_{\mathrm{j}} \lesssim 3 \times 10^{-3}$. This would be consistent with the overall low radiative efficiency of the source. We present independent arguments showing that the jet probably dominates the energetic output of all accreting black holes in the low-hard state.
\end{abstract}

Key words: accretion, accretion discs - black hole physics - magnetic field radiation mechanisms: non-thermal - star: XTE J1118+480 - X-rays: binaries

\section{INTRODUCTION}

The high energy spectrum $(>1 \mathrm{keV})$ of accreting stellar mass black holes in the low/hard state can be roughly described by a power-law with photon index $\Gamma \sim 1.4-2$, and a nearly exponential cut-off at a characteristic energy $E_{\mathrm{c}}$ of a few hundred keV (see e.g. Tanaka \& Lewin 1995; Gierlinski et al. 1997; McClintock \& Remillard 2004). Such a spectrum is generally interpreted as due to thermal Comptonisation in a plasma with electron temperature $k T_{\mathrm{e}} \sim 100 \mathrm{keV}$ and Thomson optical depth $\tau \sim 1$ (see e.g. Poutanen 1998). There are two possible explanations for the presence of this very hot plasma (often called corona). It could be either a geometrically thick, optically

* E-mail: malzac@ast.cam.ac.uk thin innermost part of the accretion flow (Shapiro, Lightman \& Eardley 1976; Narayan \& Yi 1994) or a collection of small scale active regions located atop a cold, geometrically thin and optically thick accretion disc (Haardt, Maraschi \& Ghisellini 1994), possibly powered by magnetic reconnection.

When the X-ray luminosity increases above a few percent of the Eddington luminosity $\left(L_{\mathrm{Edd}}\right)$, accreting black holes are observed to switch from the low/hard state to the so-called high/soft-state (see e.g. McClintock \& Remillard 2004). Then the X-ray luminosity is dominated by a strong thermal component originating from a geometrically thin, optically thick disc. Current observations show that the power-law component is steeper $(\Gamma \sim 2.5-3)$ and much less luminous than in the hard 
state, suggesting the disappearance of the Comptonising plasma in this state.

Recent multi-wavelength observations of accreting black holes in the hard state have shown the presence of an ubiquitous flat-spectrum radio emission (see e.g Fender 2004), that may extend up to infrared and optical wavelengths. The properties the radio emission indicate it is likely produced by synchrotron emission from relativistic electrons in compact, self-absorbed jets (Blandford \& Königl, 1979; Hiellming \& Johnston, 1988). This idea was confirmed by the discovery of a continuous and steady milliarcsecond compact jet around Cygnus X-1 (Stirling et al. 2001). Moreover, in hard state sources a tight correlation has been found between the hard X-ray and radio luminosities, holding over more than three decades in luminosity (Corbel et al. 2003; Gallo, Fender \& Pooley 2003). In contrast, during highsoft state episodes the sources appear to be radio weak (Tananbaum et al. 1972; Fender et al. 1999; Corbel et al. 2000), suggesting that the Comptonising medium of the low/hard state is closely linked to the continuous ejection of matter in the form of a small scale jet.

Merloni \& Fabian (2001) have pointed out that the energy content of the electrons in the Comptonising medium is too low to account for the observed X-ray luminosities. The Comptonising electrons have to be tightly connected to an energy reservoir where large amount of accretion power is stored before being transfered to them, and ultimately radiated. Independently, the rapid $\mathrm{X}$-ray variability in $\mathrm{Cyg} \mathrm{X}-1$ also suggests the presence of of energy reservoirs (Negoro et al. 1995; Maccarone \& Coppi 2002). Because angular momentum transport in accretion flow is most likely due to magneto-rotational instability (MRI, see Balbus \& Hawley, 1998), a natural candidate for the energy repository is the magnetic field amplified in the disc by the MRI-turbulent flow. However, if the dissipation of the tangled field (via magnetic reconnection) and non-adiabatic turbulent heating preferentially energize the protons, rather than the electrons, the hot Comptonising plasma could become twotemperature, and the protons themselves act as the main energy reservoir (Di Matteo, Blackman \& Fabian, 1997).

Models and simulations of jet production (Blandford \& Znajek 1977; Blandford \& Payne 1982; Meier 2001) indicate that jets are driven by the poloidal component of the magnetic field. Therefore storage of energy into magnetic structures driving the jet and powering the Comptonising electrons is the most straightforward explanation for the observed corona-jet association ${ }^{1}$. In this context, the corona would constitute the location where the jet is launched. These idea were developed by several authors in the context of geometrically thin and/or thick accretion flows (Meier 2001; Livio, Pringle \& King 2003) as well as accretion disc coronae (Merloni \& Fabian 2002).

Besides the radio/X-ray correlation observed on long ( $>1$ day) timescales, there are indications that the corona-jet coupling operates on timescales as short as

1 See however Markoff, Falcke \& Fender (2001) and Georganopoulos, Aharonian \& Kirk (2002) for alternative interpretations involving dominant X-ray emission from the jet. a few seconds or less. The best example is provided by the X-ray nova XTE J1118+480 (Remillard et al. 2000; McClintock et al. 2001a). During its outburst in 2000, this black hole showed all the X-ray properties of hard state sources. Fast optical and UV photometry has shown rapid optical/UV flickering presenting complex correlations with the X-ray variability (Kanbach et al. 2001; Hynes et al. 2003, hereafter K01 and H03 respectively). This correlated variability cannot be caused by reprocessing of the X-rays in the external parts of the disc. Indeed, the optical flickering occurs on average on shorter timescales than the X-ray one (K01), and reprocessing models fail to fit the complicated shape of the X-ray/optical cross correlation function (H03). Spectrally, the jet emission seems to extend at least up to the optical band (McClintock et al. 2001b; Chaty et al. 2003, hereafter C03), although the external parts of the disc may provide an important contribution to the observed flux at such wavelengths. The jet activity is thus the most likely explanation for the rapid observed optical flickering. For this reason, the properties of the optical/X-ray correlation in XTE J1118+480 might be of primary importance for the understanding of the jet-corona coupling and the ejection process.

The simultaneous optical/X-ray observations are described at length in a number of papers (K01; Spruit \& Kanbach 2001; H03; Malzac et al. 2003, hereafter M03). As discussed in these works, the observations are very challenging for any accretion model. The most puzzling pieces of evidence are the following: (a) The optical/Xray Cross-Correlation Function $(\mathrm{CCF})$ shows the optical band lagging the X-ray by $0.5 \mathrm{~s}$, but with a dip 2-5 seconds in advance of the X-rays (K01); (b) The correlation between X-ray and optical light curves appears to have timescale-invariant properties: the X-ray/optical CCF maintains a similar, but rescaled, shape on timescales ranging at least from $0.1 \mathrm{~s}$ to few $10 \mathrm{~s}$ (M03); (c) The correlation does not appear to be triggered by a single type of event (dip or flare) in the light curves; instead, as was shown by M03, optical and X-ray fluctuations of very different shapes, amplitudes and timescales are correlated in a similar way, such that the optical light curve is related to the time derivative of the X-ray one. Indeed, in the range of timescales where the coherence is maximum, the optical/X-ray phase lag are close to $\pi / 2$, indicating that the two lightcurves are related trough a differential relation. Namely, if the optical variability is representative of fluctuations in the jet power output $P_{\mathrm{j}}$, the data suggest that the jet power scales roughly like $P_{\mathrm{j}} \propto-\frac{d P_{\mathrm{x}}}{d t}$, where $P_{\mathrm{x}}$ is the $\mathrm{X}$-ray power.

Here we will show that, if indeed there is a common energy reservoir feeding both the jet and the corona, this differential relation is naturally satisfied, provided that the jet power dominates over the X-ray luminosity.

We will first present the energy reservoir model and suggest a simple physical scenario for the energy reservoir and jet disc/coupling (Section 2). We will then present a time dependent model that captures the main features of the multi-wavelength variability observed in XTE $\mathrm{J} 1118+480$, and discuss our main results obtained by comparing the properties of the simulated lightcurves with the observations (Section 3). Section 4 will be de- 
voted to a discussion of the constraints on the nature of the accretion flow in XTE J1118+480 derived from both spectral and temporal analysis, while in Section 5 we make an attempt to generalize these results to other accreting black hole sources. Finally, we summarize our conclusions in section [6]

\section{THE ENERGY RESERVOIR MODEL}

\subsection{A simple analogue}

The time-dependent model that we have developed is complicated in operation and behaviour. In order to gain some insight into its operation consider a simple model consisting of a tall water tank with an input pipe and two output pipes, one of which is much smaller than the other. The larger output one has a tap on it. The flow in the input pipe represents the power injected in the reservoir $P_{\mathrm{i}}$, that in the small output pipe the X-ray power $P_{\mathrm{x}}$ and in the large output pipe the jet power $P_{\mathrm{j}}$.

If the system is left alone the water level rises until the pressure causes $P_{\mathrm{i}}=P_{\mathrm{j}}+P_{\mathrm{x}}$. Now consider what happens when the tap is opened more, causing $P_{\mathrm{j}}$ to rise. The water level and pressure (proportional to $E$ ) drop causing $P_{\mathrm{x}}$ to reduce. If the tap is then partly closed, the water level rises, $P_{\mathrm{j}}$ decreases and $P_{\mathrm{x}}$ increases. The rate $P_{\mathrm{x}}$ depends upon the past history, or integral of $P_{\mathrm{j}}$. Identifying the optical flux as a marker of $P_{\mathrm{j}}$ and the Xray flux as a marker of $P_{\mathrm{x}}$ we obtain the basic behaviour seen in XTE J1118+480.

In the real situation we envisage that the variations in the tap are stochastically controlled by a shot noise process. There are also stochastically-controlled taps on the input and other output pipes as well. The overall behaviour is therefore complex. The model shows however that the observed complex behaviour of XTE J1118+480 can be explained by a relatively simple basic model involving several energy flows and an energy reservoir.

\subsection{A magnetic energy reservoir ?}

This simple model is largely independent of the physical nature of the energy reservoir. In a real accretion flow, the reservoir could take the form of either electromagnetic energy stored in the X-ray emitting region, or thermal (hot protons) or turbulent motions. The material in the disc could also constitute a reservoir of gravitational or rotational energy behaving as described above.

In order to be more specific, let us outline the possibility of jet disc coupling through a magnetic energy reservoir. For the sake of simplicity, we assume that the main energy reservoir for the radiating electrons is indeed the magnetic field. This would correspond to a system in which the heating of the protons by the MHD turbulence is negligible. Quataert (1998) and Quataert and Gruzinov (1999), have shown that this is the case if magnetic pressure is not much smaller than gas pressure (low plasma $\beta$ parameter), and we will therefore assume this is indeed the case here. Then, the power channelled into the particle heating (through magnetic reconnection) and escaping the corona as X-ray radiation can be written as
$P_{\mathrm{x}}=\left(v_{\mathrm{d}} / R_{\mathrm{x}}\right) E$,

where $v_{\mathrm{d}}$ is the field dissipation speed, which depends on the details of the dissipation process, $R_{\mathrm{x}}$ is the typical size of an X-ray emitting region and $E=\left(B^{2} / 8 \pi\right) V$ is the total magnetic energy contained in the hot phase $(V$ is its volume).

The jet power is instead related to the poloidal field component. For the purpose of simple estimates, the MHD jet power can be written as (Livio. Ogilvie. \& Pringle (1999)):

$P_{\mathrm{j}}=\left(B_{\mathrm{p}}^{2} / 8 \pi\right) A R_{c} \Omega$,

where $A$ is the area of the disc threaded by the poloidal field and $R_{c} \Omega$ is the typical rotational velocity of the field lines. If the field in the disc is amplified by MRI turbulence and dissipates mainly in the hot coronal phase (i.e. it does not possess a large-scale external component) , then its poloidal component can be expressed as $B_{\mathrm{p}} \simeq$ $h B$, where $h=H / R_{c}$ is the scaleheight of the hot phase (Livio et al. 1999; Meier 2001; Merloni \& Fabian 2001). The jet power can then be rewritten:

$P_{\mathrm{j}}=\frac{A}{V} h^{2} R_{c} \Omega E$.

For the sake of simplicity, we assume that the jet power is taken mainly from the tangled magnetic field. This implies that the outflow is accelerated by magnetic dissipation processes and/or that the magnetic energy of the corona is carried away by an essentially electromagnetic outflow. In fact, it is possible that the jet is powered directly by the disc rotational energy without significant field dissipation. This would be the case whenever the poloidal field is coherent on large enough scales to exert a significant torque on the disc (see e.g. the model of King et al. 2003). However, such a mechanism for angular momentum transport in the disc competes with MRI, so that when large scale fields extract energy and angular momentum from the disc, the MRI dynamo switches off, also draining the magnetic energy reservoir.

Summarizing, we will make the assumption that both the jet and X-ray power are tapped from the same magnetic energy reservoir. Note that from equations 1 and 3 both jet and X-ray powers scale linearly with the total reservoir energy. The total power extracted from the magnetic field in the hot phase is then:

$P_{\mathrm{x}}+P_{\mathrm{j}}=\frac{B^{2}}{8 \pi}\left(\frac{V v_{\mathrm{d}}}{R_{\mathrm{x}}}+A h^{2} R_{c} \Omega\right)$.

If we define the relative fractional power of the jet $\eta=P_{\mathrm{i}} /\left(P_{\mathrm{x}}+P_{\mathrm{j}}\right)$, we then find that (see also Merloni \& Fabian, 2002)

$\eta=\left(1+\frac{V}{A R_{\mathrm{x}}} \frac{v_{\mathrm{d}}}{R_{c} \Omega} h^{-2}\right)^{-1}$.

The discussion so far makes no distinction between a corona made of a collection of active phases atop a cold disc and a continuous hot inner flow. Indeed the differences show up in the geometrical factors in the above equations. In the case of a structured corona, we consider $N$ cylindrical active region (magnetic tubes) located atop the disc at distances ranging from $3 R_{\mathrm{S}}$ to $R_{\mathrm{c}}$. The typical radius $R_{a}$ and height $H_{a}$ of the active regions 
scale linearly with their the distance $R$ from the black hole, we note $a=R_{a}(R) / R$ and $h=H_{a}(R) / R$. We assume that their radial distribution scales like $1 / R$ so that the covering factor of the corona is independent of distance. Then the average radius of an active region is $R_{\mathrm{x}}=a R_{\mathrm{c}} / \ln \left(R_{\mathrm{c}} / 3 R_{\mathrm{S}}\right), A=(\pi / 2) N a^{2} R_{\mathrm{c}}^{2} / \ln \left(R_{\mathrm{c}} / 3 R_{\mathrm{S}}\right)$, and $V=(\pi / 3) N a^{2} h R_{\mathrm{c}}^{3} / \ln \left(R_{\mathrm{c}} / 3 R_{\mathrm{S}}\right)$.

On the other hand, in the thick disc case, we have $R_{\mathrm{x}}=R_{c}, A=\pi R_{\mathrm{c}}^{2}$ and $V=(2 / 3) \pi h R_{\mathrm{c}}^{3}$, where here $R_{\mathrm{c}}$ is taken to coincide with the outer radius of the thick disc and a constant $H / R$ (wedge-like geometry) is assumed.

\subsection{Time dependent model}

In a stationary flow, the extracted power $P_{\mathrm{j}}+P_{\mathrm{x}}$ would be perfectly balanced by the power injected into the magnetic field, which is, in the most general case, given by the difference between the accretion power and the power advected into the hole and/or stored in convective motions $^{2}: P_{i} \simeq \dot{M} c^{2}-P_{\text {adv,conv }}$. However, observations of strong variability on short time scale clearly indicate that the heating and cooling of the X-ray (and optical) emitting plasma are highly transient phenomena, and the corona is unlikely to be in complete energy balance on short timescales. We therefore introduce a timedependent equation governing the evolution of its total energy $E$ :

$\dot{E}=P_{\mathrm{i}}-P_{\mathrm{j}}-P_{\mathrm{x}}$,

and we assume that all the three terms on the right hand side are time dependent.

Furthermore, we assume that the optical light comes mainly from synchrotron emission in the inner part of the jet. A contribution from X-ray light reprocessed in the external part of the disc could be present in the optical emission. However, this component is likely to be weak (cf. K01; H03).

The (time averaged) total observed optical flux is:

$O_{p t} \propto P_{\mathrm{j}}+f_{\mathrm{r}} P_{\mathrm{x}}$

where the normalization factor $f_{\mathrm{r}}$ depends on the geometry of the system and on the optical wavelength, with $f_{\mathrm{r}}$ increasing towards shorter wavelengths ${ }^{3}$.

2 In fact, the secular evolution of Convection Dominated Accretion Flow leads to accumulation of mass at the outer disc boundary, and thus to a non stationary flow. However, if we limit ourselves to observations which are shorter than a typical outburst duration, we can regard convection as an alternative escape route for the graviational energy dissipated by the accreting matter out of the region of interest (where X-ray and optical emission are produced).

3 In the fully time dependent model we will consider, additional effects on the properties of the optical emission should be considered: First of all there is a time delay $\Delta$ between matter ejection and dissipation in the form of optical photons. As the jet is relativistic and the optical light is produced at short distance $\left(\lesssim 1000 G M / c^{2}\right)$ from the hole, the delay should be $\Delta \lesssim 0.1 \mathrm{~s}$. Similarly, reprocessing will also introduce a time delay of order of $0.1 \mathrm{~s}$ and any variability of $P_{\mathrm{x}}$ on time scales shorter than a few seconds will not be apparent in the reprocessed light
We introduce the instantaneous dissipation rates $K_{\mathrm{j}}$ and $K_{\mathrm{x}}$ :

$P_{\mathrm{j}}(t)=K_{\mathrm{j}}(t) E(t)$

$P_{\mathrm{x}}(t)=K_{\mathrm{x}}(t) E(t)$,

and we consistently denote $\left\langle K_{\mathrm{j}}\right\rangle,\left\langle K_{\mathrm{x}}\right\rangle,\left\langle P_{\mathrm{i}}\right\rangle$, and $\langle E\rangle$ as the time averaged values. We define the average dissipation time:

$T_{\text {dis }}=\left(\left\langle K_{\mathrm{j}}\right\rangle+\left\langle K_{\mathrm{x}}\right\rangle\right)^{-1}$,

such that if the energy reservoir is not fed (i.e. $P_{\mathrm{i}}=0$ ) its level decays with an e-folding time $T_{\text {dis }}$. Also, it is convenient to define

$f_{\mathrm{x}}=1-\eta=\left\langle K_{\mathrm{x}}\right\rangle T_{\mathrm{dis}}$,

which is the average fraction of the total power that goes into the X-ray emission. In the framework of the magnetic reservoir of section 2.2 the average values of the dissipation rates are related by eqs. (1) and (2) to the (time time averaged) values of the physical parameter of the system. Namely: $\left\langle K_{\mathrm{j}}\right\rangle=\left(A h^{2} / V\right) R_{\mathrm{c}} \Omega$ and $\left\langle K_{\mathrm{x}}\right\rangle=v_{\mathrm{d}} / R_{\mathrm{x}}$.

Obviously, the detailed physical modeling of the time evolution of such a jet corona system is an extremely complex problem that is beyond the present computer capabilities. Instead we will adopt a more phenomenological approach. We will model the variability of the source by assuming random fluctuations of $K_{\mathrm{j}}, K_{\mathrm{x}}$ and $P_{\mathrm{i}}$, then compare the results with the observations and constrain the properties of these fluctuations.

In general, both the instantaneous injected power $P_{\mathrm{i}}$ and the dissipation rates $K_{\mathrm{j}}$ and $K_{\mathrm{x}}$ may depend on the amount of energy $E$ stored in the reservoir. However, the fact that $P_{\mathrm{i}}$ and $K_{\mathrm{x}}$ are required to vary on time scales different from those of $P_{\mathrm{x}}$ (see below), suggest that they are rather independent of $E$. Moreover, observations show that in black hole binaries and Seyfert galaxies the X-ray amplitude of variability is linearly related to the X-ray flux level (Uttley \& Mc Hardy 2001). In general, if $P_{\mathrm{i}}$, $K_{\mathrm{x}}$ and $K_{\mathrm{j}}$ are either strongly dependent on $E$, or correlated with each other, this would introduce a non-linear relation between RMS amplitude and observed flux. The observations thus suggest that the values of $P_{\mathrm{i}}$ and the dissipation rates $K_{\mathrm{j}}$ and $K_{\mathrm{x}}$ at a given time are nearly independent of the level of the reservoir energy (although $E$ does depend on the history of $K_{\mathrm{j}}$ and $K_{\mathrm{x}}$ ). For these reasons, as well as for the sake of simplicity, we will then assume that $K_{\mathrm{j}}, K_{\mathrm{x}}$ and $P_{\mathrm{i}}$ fluctuate randomly and independently, driving the fluctuations of the energy reservoir.

For the specific form of the fluctuation, we use exponentially rising shot profiles:

$s(t)=A \frac{\exp (t / \tau)-1}{\exp (1)-1} \quad$ for $\quad t<\tau ;$

This profile was chosen for its simplicity. Our results regarding the optical/X-ray correlation are not sensitive to the shape of the individual flares. On the other hand they will strongly depend on the amplitude, time-scales and occurence rate of the shots. The amplitude $A$ and the occurrence rate $\lambda$ of the random shots are taken constant. These quantities are related to the average dissipation rate. The shot duration $\tau$ is distributed within 
$\tau_{\min }$ and $\tau_{\max }$ with a power law distribution $\rho(\tau) \propto \tau^{-p}$. These parameters constrain the fractional amplitude of variability and the shape of the power spectrum of the fluctuations that are imposed on the system. The fractional RMS scales like $1 / \sqrt{\lambda}$, and the power spectrum is a power law ranging from frequencies $1 / \tau_{\max }$ to $1 / \tau_{\min }$ with a slope $\alpha=3-p$ (see e.g. Poutanen and Fabian 1999). For $K_{\mathrm{x}}, K_{\mathrm{j}}$ and $P_{\mathrm{i}}$, the parameters $\lambda, p, \tau_{m i n}$, $\tau_{\max }$ are, in general, not identical. In presenting our results, the subscripts $x, j$ and $i$ will refer to $K_{\mathrm{x}}, K_{\mathrm{j}}$ and $P_{\text {i }}$ respectively.

For a specific set of parameters we first generate time series for $K_{\mathrm{x}}, K_{\mathrm{j}}$ and $P_{\mathrm{i}}$, solve the time evolution of the energy reservoir $E$ and then use it to derive the the resulting optical and X-ray light curves. The ejected material travels from the corona to the shock region or photosphere where the optical photons are produced. As discussed above, this introduces a time delay of $\Delta<0.1 \mathrm{~s}$ in the optical emission. To model this delay, we simply shift the optical light curve by $\Delta$. We then compute the X-ray and optical Power Density Spectra (PDS), Auto Correlation Functions (ACF), their Cross-Correlation Function (CCF), coherence and phase lag spectrum for comparison with the observed ones, in order to obtain the largest possible number of different observational tests for our variability model.

\subsection{Main observational constraints}

Before we proceed and illustrate the results of our simulations, a remark is in place with respect to the requirement any specific realization of our model will have to fulfill in order to reproduce the main observational characteristics. This will guide us in the exploration of the vast parameter space of the model, as well as provide us with useful insight on the physical interpretation of the results.

We first note that combining equations (6) and (9) we obtain for the total instantaneous jet power the following relation:

$P_{\mathrm{j}}=P_{\mathrm{i}}-\left(1+\frac{\dot{K}_{\mathrm{x}}}{K_{\mathrm{x}}^{2}}\right) P_{\mathrm{x}}-\dot{P}_{\mathrm{x}} / K_{\mathrm{x}}$

We can see from this equation that the differential scaling $P_{\mathrm{j}} \propto-\dot{P}_{\mathrm{x}}$, observed in XTEJ1118+480, will be rigorously reproduced provided that:

- $K_{\mathrm{x}}$ is a constant;

- $P_{\mathrm{i}}-P_{\mathrm{x}}$ is a constant. ${ }^{4}$

It is physically unlikely that those conditions will be exactly verified. In particular, $P_{\mathrm{x}}$ is observed to have a large RMS amplitude of variability of about 30 percent. However, the observed differential relation holds only roughly and only for fluctuations within a relatively narrow range of time-scales $1-10 s$. Therefore, the above conditions need only to be fulfilled approximatively and for low frequency fluctuations $(>1 \mathrm{~s})$. In practice, the

4 This constant can differ from 0 because the differential scaling is observationally demonstrated only for the varying fraction of the optical and X-ray fluxes. following requirements will be enough to make sure that the low frequency fluctuations of the right hand side of equation 13 are dominated by $\dot{P}_{\mathrm{x}}$ :

- $P_{\mathrm{x}} \ll P_{\mathrm{i}}$, implying that the jet power, on average, dominates over the X-ray luminosity;

- the amplitude of variability of $K_{\mathrm{x}}$ and $P_{\mathrm{i}}$ in the 1-10 s range is low compared to that of $P_{\mathrm{j}}$. In other words the 1-10 s fluctuations of the system are mainly driven by the jet activity.

The first condition is crucial, and will be discussed in more detail in Section 4 The second condition requires that the mechanisms for energy reservoir filling and dissipation in the corona and in the jet are occurring on quite different timescales, and this will also provide us with additional constraints on the global dynamical properties of the system.

\section{RESULTS}

In figure 1 we show the results of a simulation with $T_{\text {dis }}=0.5 \mathrm{~s}$ and $f_{\mathrm{x}}=0.1$ (see Table 1 for the value of the other parameters). The model produces an X-ray power spectrum with a plateau up to $\sim 0.1 \mathrm{~Hz}$ and a powerlaw component with slope $\sim 1.4$ above that frequency, with most of the X-ray variability occurring around 0.1 Hz. The optical PDS power-law has a flatter slope $(\sim 1)$ up to $1 \mathrm{~Hz}$ and then softens to a slope similar to that of the X-ray PDS. The resulting optical ACF is significantly narrower than the X-ray one. The full-width-athalf-maximum (FWHM) of the two ACFs differs by a factor $>2$. The overall coherence is low $(<0.4)$ : reaching a maximum in the $0.1-1 \mathrm{~Hz}$ range and decreasing rapidly both at lower and higher frequency. The phase-lags are close to $\pi / 2$ in the $0.1-1 \mathrm{~Hz}$ range and increase from 0 at low frequencies up to $\pi$ at around $6 \mathrm{~Hz}$. At higher frequencies the phase lags spectrum is characterized by large oscillations. Finally the resulting CCF rises very quickly at positive optical lags, peaks around $0.5 \mathrm{~s}$ (this is the post-peak) and then declines slowly at larger lags. The two bands appear to be anti-correlated at negative optical lags indicating a systematic optical dip 1-2 s before the X-rays reach their maximum (pre-dip).

All these characteristics are observed in XTE J1118+480. Moreover, the integrated X-ray RMS is 25 percent as observed. The optical variability is 20 percent slightly larger than the 16 percent reported by M03 but this could be reduced if the expected constant disc component was added.

Obviously the model parameter values were carefully selected in order to reproduce these characteristics. Given the complexity of the model, a full investigation of the parameter space is premature. However, it may be useful to illustrate the main effects of the different parameters by considering several simple situations. 


\subsection{Variability dominated by the jet dissipation rate $K_{\mathrm{j}}$}

\subsubsection{Jet dominated models $\left(f_{\mathrm{x}}<<1\right)$}

We first consider the case where the X-ray emission is energetically negligible. The jet fully drives the variability of the system in the limit of large $\lambda_{\mathrm{i}}$ and $\lambda_{\mathrm{x}}$, and low $f_{\mathrm{x}}$. In this limit, both conditions for the applicability of the model discussed in the previous section are exactly verified. We thus expect the scaling $P_{\mathrm{j}} \propto-\dot{P}_{\mathrm{x}}$ to be realised. Indeed, we obtain phase lags that are close to $\pi / 2$ independent of frequency. In this limit, the resulting $\mathrm{CCF}$ is purely anti-symetric with pre-dip and post-peak of identical amplitude and lag (contrary to what is observed). The optical lag of the post-peak is controled by $T_{\text {dis }}$, which sets the time scale on which the energy reservoir responds to the imposed fluctuations. At frequencies larger than $1 / T_{\text {dis }}$ the $\mathrm{X}$-ray variability decreases because there cannot be any variability of the reservoir energy $E$ on time scales larger than $T_{\text {dis }}$. The coherence function also decreases at frequencies above $1 / T_{\text {dis }}$. On the other hand, at frequencies lower than $1 / T_{\text {dis }}$ the optical variability decreases because at such low frequencies the system evolves in a quasi-static way. Since $P_{\mathrm{i}}$ is constant and $P_{\mathrm{x}}$ is energetically negligible, the variability of $K_{\mathrm{j}}$ is compensated by the variability of $E$ so that $P_{\mathrm{j}}$ remains almost constant, too.

\subsubsection{X-ray dominated models $\left(f_{\mathrm{x}} \sim 1\right)$}

If $f_{\mathrm{x}}$ is large but the amplitude of variability of $K_{\mathrm{x}}$ and $P_{\mathrm{i}}$ are kept negligible by increasing $\lambda_{\mathrm{x}}$ and $\lambda_{\mathrm{i}}$, then the power output can be dominated by the X-rays but the variability is still driven by the jet.

In this case, we still have $\pi / 2$ phase-lags but only at frequencies $>1 / T_{\text {dis }}$. The effects of a large X-ray dissipation is the appearance of phase-lags $\pi$ at frequencies $<1 / T_{\text {dis }}$ indicating an anti-correlation. This anticorrelation is also apparent in the CCF, which tends to be negative and symmetric around zero lag. The anticorrelation at low frequencies is due to the fact that on long time-scales the system is always in equilibrium, but contrary to the jet dominated case, X-ray losses are not negligible in this case. The reservoir energy $E$ changes in order to keep the total output constant (and equal to $P_{\mathrm{i}}$ ), but $P_{\mathrm{j}}$ does change. For this reason the fluctuations of the jet output are strictly anti-correlated to that of the X-ray power. Such an anti-correlation is in clear conflict with the data, the observed phase-lags indicating rather a correlation at low frequencies. Thus, even if the jet drives the variability as in the previous example, we definitely need $f_{\mathrm{x}} \ll 1$ in order to reproduce the data. In practice, exploring a large volume of the parameter space we could not find a reasonable agreement with the data for $f_{\mathrm{x}}$ larger than $\sim 0.2$. This is a very robust result of our time-dependent modeling, and we will examine its consequences in Section 4

\subsection{Variability dominated by the coronal dissipation rate $K_{\mathrm{x}}$ and/or by the power input $P_{\mathrm{i}}$}

The situation where the variability is dominated by the $\mathrm{X}$-ray dissipation rate in the corona is perfectly symmetric to the case where the variability is dominated by the jet, studied above, with however an inversion of the sign of phase lags and time-axis in the CCF plot.

On the other hand, fluctuations of $P_{\mathrm{i}}$ introduce perfectly positively correlated fluctuations of $P_{\mathrm{j}}$ and $P_{\mathrm{x}}$. If the fluctuations of $P_{\mathrm{i}}$ dominate we obtain X-ray and optical light curves that are perfectly correlated on all time scales with identical power spectrum and unity coherence, in contrast with the observations.

In general, when in a given range of timescales more than one component (i.e. $P_{\mathrm{i}}, K_{\mathrm{j}}$ or $K_{\mathrm{x}}$ ) drives the variability, the main effect is a strong reduction of the coherence function in the corresponding range of frequencies.

\subsection{A realistic case}

Now that we understand the basic effects of the different parameters, we get back to our 'realistic' model and explain how we were guided toward this solution and how it reproduces qualitatively all the timing features observed in XTE J1118+480.

As discussed above, reproducing the data requires the jet to dominate the energy budget and be the main driver of the variability (at least for the 1-10 s fluctuations). Therefore we set $f_{\mathrm{x}}=0.1$ and the parameters $\lambda_{j}$, $p_{j}, \tau_{\min j}$ and $\tau_{\max j}$ were adjusted in order to have large enough 1-10 s fluctuations of the jet power. These parameters also control the shape of the optical power spectrum. In particular, the slope of the shot distribution $p_{j}$ is chosen so that it leads to the observed slope of the optical power spectrum above $1 \mathrm{~Hz}$. The dissipation time is set to $T_{\text {dis }}=0.5$ in order to match the observed optical lag in the CCF reported by K01.

The values of $\lambda_{i}, p_{i}, \tau_{\min i}$ and $\tau_{\max i}$ were fixed so that the variability of $P_{\mathrm{i}}$ introduces some correlated variability at low frequencies, enabling us to reproduce the asymmetry of the CCF and the small phase-lags observed at low frequency. The loss of coherence below $10 \mathrm{~Hz}$ is due to the simultaneous large variability of $P_{\mathrm{i}}$ and $K_{\mathrm{j}}$ at low frequencies.

Finally, we also introduce fluctuations of the X-ray dissipation rate at high frequencies. This enables us to reproduce the loss of coherence observed above one $\mathrm{Hz}$. Also $\lambda_{x}, p_{x}, \tau_{\min x}$ and $\tau_{\max x}$ were chosen in order to reproduce the X-ray power spectrum at high frequencies which is otherwise too steep.

A propagation lag of $\Delta=0.05 \mathrm{~s}$ then provides the observed high frequency behaviour of the phase lags. If the propagation lag is neglected, the phase lag spectrum is flat at high frequencies with phase lag at a constant value $\sim \pi / 2$. The constant time delay introduces an additional phase lag $\psi=2 \pi f \Delta$. As the time-scale of the fluctuations approaches that of the time delay this additional phase lag becomes dominant. The overall phase lag thus increases and reaches $\pi$ at $\sim 6 \mathrm{~Hz}$. The phase lags are defined only in the range $[-\pi, \pi]$ and can be 
only measured modulo $2 \pi$. Thus, at higher frequencies the phase lag shifts to $-\pi$ then increases linearly to $\pi$ and so on, producing large 'oscillations' in the pahse-lag spectrum. A similar behaviour of the phase lags above $1 \mathrm{~Hz}$ is observed in XTE J1118+480 (see M03, Fig. 5), indeed suggesting a propagation lag of $\Delta \sim 0.05 \mathrm{~s}$. On the other hand, the overall effects of propagation delays on the $\mathrm{CCF}$ and coherence function are negligible.

\subsection{The effects of reprocessing}

We now consider the effects of a possible reprocessing component from the disc. We model this component by convolving the X-ray lightcurve with a transfer function $T_{r}$ describing the time delay and variability smearing due to reprocessing. We use the following transfer function :

$$
\begin{aligned}
T_{r}(t) & \propto \frac{t}{\delta} \exp (-t) \exp \left[\left(\frac{t}{\delta}\right)^{0.01}-\frac{t}{\delta}\right] \\
& +0.28\left[1-\exp \left(-10 \frac{t}{\delta}\right)\right] \exp \left[-(t / 5)^{20}\right]
\end{aligned}
$$

where $t$ is measured in seconds and the reprocessing time delay $\delta=0.1 \mathrm{~s}$.

This function is a rough analytic approximation to the theoretical transfer functions shown in H03.

The reprocessed component is added to the optical light curve. Its normalization is parametrized by the ratio of the average flux of the reprocessed component to that of the jet component. In the simulation shown in Figure2 we take this ratio to be 0.5 , keeping all other parameters' values identical to those of the previous simulation. The main effect is to add a low frequency component to the optical lightcurves highly correlated to the X-ray one. By comparison with Figure 1 the optical ACF is broader, the coherence is higher at low frequency and the optical pre-dip tends to disappear, the optical lags are shorter in particular at low frequency.

The increase of the reprocessed component is associated with an increase in the coherence function, and the X-ray and optical ACF and PDS becoming similar and shorter optical lags. We expect to observe this evolution if we look at shorter and shorter wavelengths where the disc emission increasingly dominates the total optical/UV flux. Indeed H03 report similar trends in the dependence of the correlation upon wavelength. We also note that at low frequencies $(<0.1 \mathrm{~Hz}), \mathrm{H} 03$ observe a large coherence in the far UV domain, while M03 report almost zero coherence. This could be interpreted as the effects of reprocessing in the far UV that would become negligible at optical wavelengths.

\section{THE NATURE OF THE LOW-LUMINOSITY ACCRETION FLOW IN XTE J1118+480}

\subsection{Constraints from the time variability study}

The detailed analysis of the complicated structure of the multi-wavelength variability of the source have allowed us to select a region of parameter space for which our model successfully reproduces all the main observational

\begin{tabular}{ccc}
\hline$T_{\text {dis }}=0.5 \mathrm{~s}$ & $f_{\mathrm{x}}=0.1$ & \\
$\lambda_{\mathrm{i}}=100 \mathrm{~s}^{-1}$ & $\lambda_{\mathrm{j}}=50 \mathrm{~s}^{-1}$ & $\lambda_{\mathrm{x}}=1000 \mathrm{~s}^{-1}$ \\
$p_{\mathrm{i}}=2.1$ & $p_{\mathrm{j}}=1.4$ & $p_{\mathrm{x}}=1.1$ \\
$\tau_{\min i}=0.1 \mathrm{~s}$ & $\tau_{\min j}=0.01 \mathrm{~s}$ & $\tau_{\min x}=0.01 \mathrm{~s}$ \\
$\tau_{\max i}=7 \mathrm{~s}$ & $\tau_{\max j}=10 \mathrm{~s}$ & $\tau_{\max x}=0.5 \mathrm{~s}$ \\
\hline RMS $P_{\mathrm{i}}=0.20$ & $K_{\mathrm{j}}=0.27$ & $K_{\mathrm{x}}=0.11$ \\
RMS $E=0.23$ & $\mathrm{Opt}=0.21$ & $X=0.26$ \\
\hline
\end{tabular}

Table 1. The model parameters used in the simulations of Fig. 11 The two last rows show the resulting fractional amplitude of the dissipation rates, energy reservoir, optical and $\mathrm{X}$-ray fluxes. In model 2 some reprocessing was added and the optical fractional RMS is then reduced to 0.16 .

pieces of evidence. The parameters are summarized in Table 1 In principle these parameters can contrain the dynamic and geometry of the accretion flow. However this would require a detailed physical model for the jet/disc coupling. Here we will assume that, as suggested in section 2.2 the magnetic field constitutes the main energy reservoir and investigate the consequences for the accretion flow.

Let us start by examining the two parameters that reflect the time-averaged global energetics of the system: $T_{\text {dis }}$ and $f_{\mathrm{x}}$. From Eqs. (1), (3), (10) and (11), we see that they are related to the physical parameters of the system:

$\frac{v_{\mathrm{d}}}{R_{\mathrm{x}}}=\frac{f_{\mathrm{x}}}{T_{\mathrm{dis}}}$

$\frac{A}{V} h^{2} v_{\mathrm{K}}\left(R_{\mathrm{c}}\right)=\frac{1-f_{\mathrm{x}}}{T_{\mathrm{dis}}}$,

where we have assumed that the disc rotation law is Keplerian and we denote $v_{\mathrm{K}}\left(R_{\mathrm{c}}\right)$ the Keplerian speed at the radius $R_{\mathrm{c}}$.

In the estimates below, we will assume, as we do throughout the paper, a black hole mass of $10 M_{\odot}$ for XTE J1118+480. Equation (15) is a constraint on the magnetic field dissipation speed in the hot phase: for $f_{\mathrm{x}}=$ 0.1 and $T_{\text {dis }}=0.5$ it can be rewritten as:

$\frac{v_{\mathrm{d}}}{c} \simeq 2 \times 10^{-5} r_{\mathrm{x}}$,

where $r_{\mathrm{x}}=R_{\mathrm{x}} / R_{\mathrm{S}}$.

On the other hand, Equation (16) constrains the corona/hot flow scaleheight, which should be defined by selecting one of the two possibility for the hot phase geometry. It turns out that for both geometries we obtain the same relation:

$h \simeq 1.7 \times 10^{-1} \frac{0.5}{T_{\text {dis }}} \frac{\eta}{0.9}\left(\frac{r_{\mathrm{c}}}{100}\right)^{3 / 2}$

where $r_{\mathrm{c}}=R_{\mathrm{c}} / R_{\mathrm{S}}$.

In the case of the thick inner flow, self-consistency would require a substantial scaleheight of the hot flow. If, for example, we require $h \gtrsim 0.1$, we obtain a constraint on the radial extent of the inner hot flow $r_{\mathrm{c}} \gtrsim 70$, which, incidentally, appear to be consistent with the spectroscopically-inferred inner radius of the truncated cold disc (Esin et al. 2001; Chaty et al. 2003).

The same spectroscopic constraints also apply to the patchy corona case (see next section) and the radial extension of the corona above the disc should also be at 

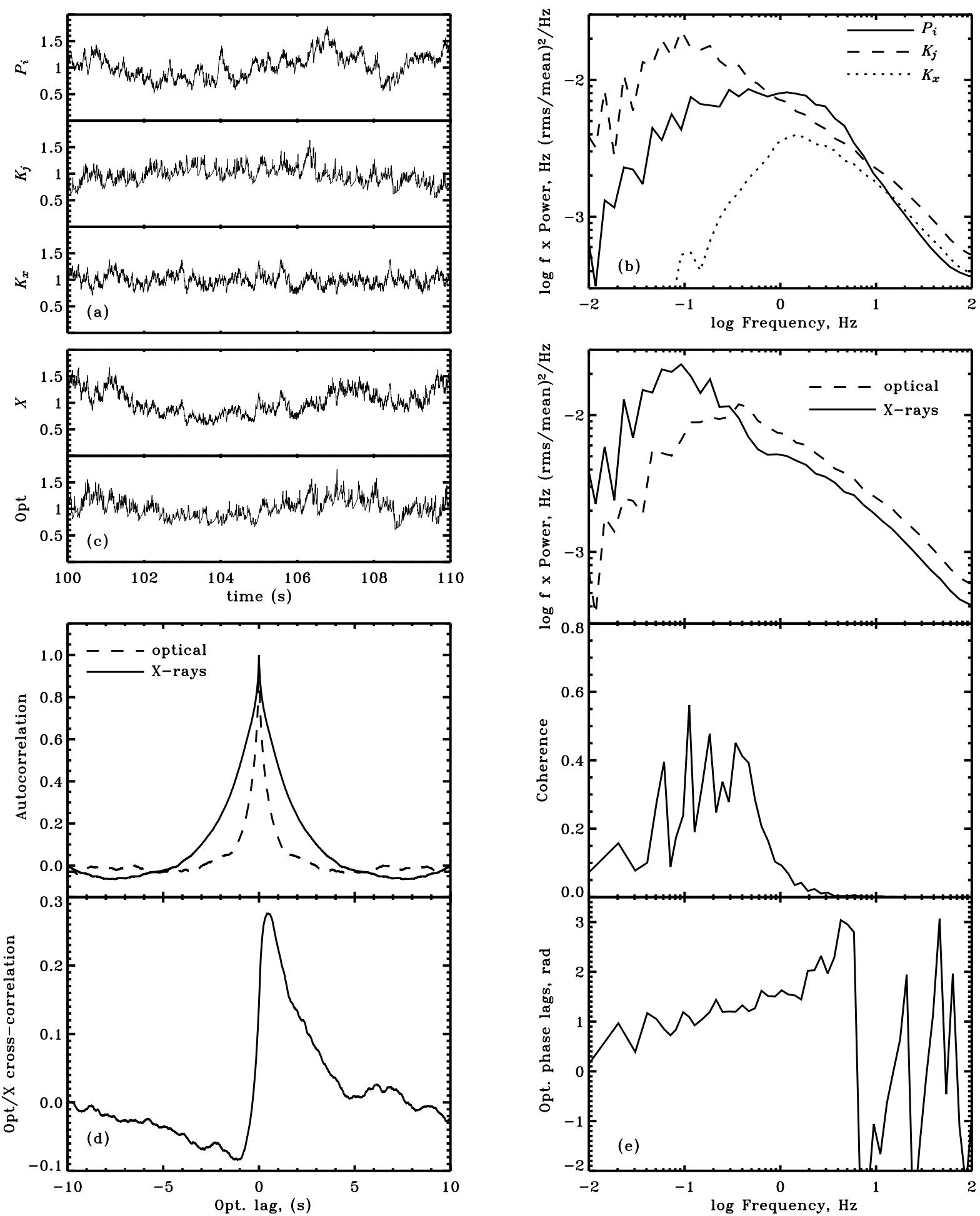

Figure 1. Results of simulations for parameters given in table 1 Sample time series (panel a) and power spectra (panel b) of $P_{\mathrm{i}}, K_{\mathrm{j}}, K_{\mathrm{x}}$, resulting X-ray and optical fluxes light curves (panel c), X-ray/optical autocorrelation and cross-correlation functions (panel d), power spectra, coherence and phase-lags (panel e). 

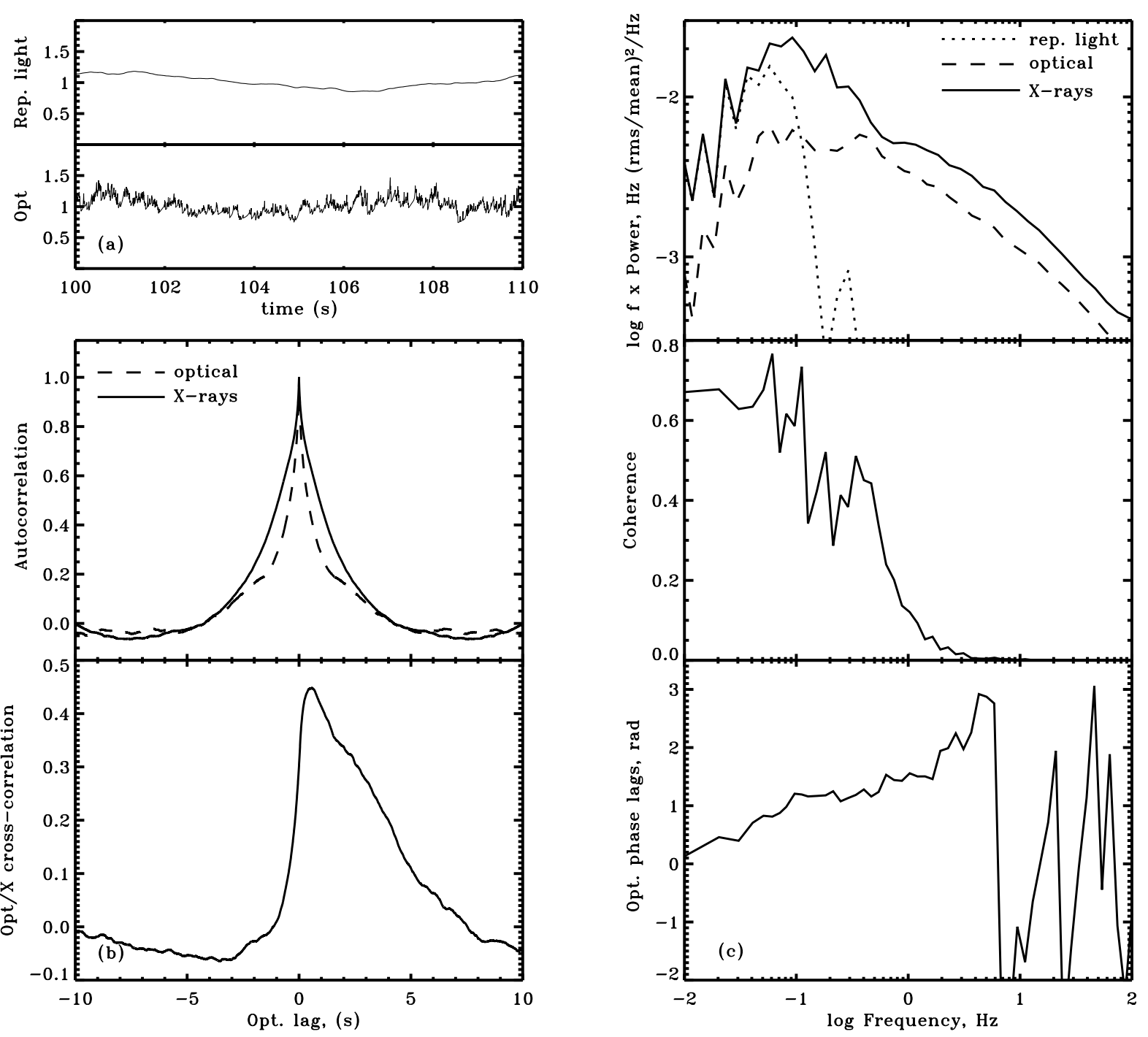

Figure 2. Same simulation as in Fig. 1 but adding a disc reprocessing component accounting for 30 percent of the optical flux on average (see section 3.4. Panel a: sample light curves of the reprocessed component and total optical Panel b: X-ray/optical autocorrelation and cross-correlation functions. Panel c: power spectra of the reprocessed component, total optical and X-rays (top), X-ray/optical coherence and phase-lags (bottom).

least $r_{\mathrm{c}} \sim 100$. Then for typical inferred values of the size of the active regions $\left(r_{\mathrm{x}} \sim\right.$ a few, see e.g Haardt, Maraschi \& Ghisellini 1994; Di Matteo, Celotti \& Fabian 1999), the aspect ratio of the cylindrical regions writes:

$\frac{h}{a} \simeq 1.6 \frac{0.5}{T_{\mathrm{dis}}} \frac{\eta}{0.9} \frac{3}{r_{\mathrm{x}}}\left(\frac{r_{\mathrm{c}}}{100}\right)^{5 / 2} \frac{\ln \left(r_{\mathrm{c}} / 3\right)}{\ln (100 / 3)}$.

It appears to be slightly larger than unity, as required to reproduce the hard X-ray spectrum (see e.g. Malzac, Beloborodov \& Poutanen 2001). Note that the aspect ratio is very sensitive to the coronal radius. If $r_{\mathrm{c}}$ is as large as 350, as inferred by Chaty et al. (2003) we obtain a quite large ratio $h / a \simeq 27$, then internally generated cyclosynchrotron radiation should dominate over reprocessing as a source of seed photons for the Comptonisation process.

Moreover, combining the expression that relates the observed X-ray luminosity (1) with the constraints ob- tained above on the field dissipation speed, we can estimate the value of the magnetic field. In the case of the thick inner flow we obtain:

$B \simeq 3.2 \times 10^{6}\left[\frac{f_{\mathrm{x}}}{0.1} \frac{\eta}{0.9}\right]^{-1 / 2} \frac{T_{\mathrm{dis}}}{0.5 \mathrm{~s}}\left(\frac{r_{\mathrm{c}}}{100}\right)^{-9 / 4} \mathrm{G}$

From the lower limit on the inner disc radius derived above, we conclude that $B \lesssim 7 \times 10^{6} \mathrm{G}$. In the case of the patchy corona:

$$
\begin{gathered}
B \simeq 2.5 \times 10^{7}\left[\frac{f_{\mathrm{x}}}{0.1} \frac{\eta}{0.9} \frac{\ln \left(r_{\mathrm{c}} / 3\right)}{\ln (100 / 3)} \frac{N}{10}\right]^{-1 / 2} \\
\frac{T_{\mathrm{dis}}}{0.5 \mathrm{~s}} \frac{3}{r_{\mathrm{x}}}\left(\frac{r_{\mathrm{c}}}{100}\right)^{-5 / 4} \mathrm{G}
\end{gathered}
$$

We note that the RMS variability amplitude should scale as $N^{-1 / 2}$, so that the magnetic field intensity is directly proportional to the observed variability level.

Finally, let us discuss the different variability 
timescales of the dissipation rates, as fixed in our fiducial model. On the one hand, the range of timescales on which the variability of $K_{\mathrm{x}}$ is maximal are between 50 and $1 \mathrm{~Hz}$, corresponding to the Keplerian frequencies between 10 and $100 R_{S}$ (for a $10 M_{\odot}$ black hole). This is consistent with the X-rays being produced in the hot phase of the accretion flow (either in a geometrically thick disc or in the corona). In general the dissipation of the tangled magnetic field into particle heating is essentially a local process acting on timescales comparable to the local dynamical time. Therefore the minimum and maximum variability timescales of $K_{\mathrm{x}}$ reflect the smallest and largest radii where we have substantial energy in the hot phase/corona.

On the other hand, we see from Figure 1 that the variability of $K_{\mathrm{j}}$ is high on much wider range of timescales than $K_{\mathrm{x}}$, from 0.01 to 10 seconds. As discussed in sections 2.4 and 3 the data require that at low frequencies the fluctuations of $K_{\mathrm{j}}$ dominate over the other sources of variability. The physical interpretation is that generating a powerful outflow requires large-scale coherent poloidal field structures threading a significant fraction of the inner disc surface and extending also in the vertical direction. Such structures may take more time to build and destroy. So, locally, the timescale of variation of $K_{\mathrm{j}}$ can go from the dynamical time itself to larger timescales (in the rare cases where the field builds up its poloidal component to a larger scale). Based on simple arguments, Livio, Pringle \& King (2003) estimate that the time-scale for establishing a change in the scale of the poloidal component of the magnetic field through dynamo processes is larger than the dynamical time scale by a factor $t_{j e t} / t_{d y n} \sim 2^{1 / h}$. Since our model indicates $t_{\text {jet }} / t_{\text {dyn }} \sim 10$, this would imply $h \sim 0.3$, which appears to be in agreement with the independent estimate provided by equation 18

We also note that the variability of $K_{j}$ induces large amplitude fluctuations of the energy reservoir that in turn lead to an important X-ray variability on time-scales much larger than the dynamical time. In fact, most of the observed X-ray variability is caused by the jet activity, providing an explanation for the long observed timescales. Recently King et al. (2003) have proposed a detailed physical model for the X-ray variability of accreting black holes that considers a similar situation where largescale ejection events modulate the strong X-ray emission from the inner disc.

\subsection{Constraints from time averaged spectroscopy}

We have shown in section 3 that there is an important condition for our a model to work, namely that the jet power should dominate over the X-ray luminosity. We will now discuss how realistic this assumption is.

First, let us examine the observed energetic output of XTE J1118+480. We stress that this is the best source available for such kind of study, given its unsurpassed spectral coverage. In the following we will express all the luminosities in units of the Eddington luminosity $\left(L_{\mathrm{Edd}}=1.3 \times 10^{38}\left(M / M_{\odot}\right) \mathrm{erg} \mathrm{s}^{-1}\right.$, with $\left.M=10 M_{\odot}\right)$.
The numbers given below are taken from the results of the multi-wavelength spectral analysis of Chaty et al. (2003). These authors decomposed the overall SED into three components:

- The jet component has a total observed radiative luminosity of $L_{\mathrm{j}}=3.8 \times 10^{-5}$, assuming isotropy and integrating the power-law emission from the radio to the optical band. In the optical/UV the emission should start to be dominated by the cold accretion disc, but the possibility that the jet spectrum extends up into the UV domain cannot be ruled out. Indeed, the fact that H03 observe similar correlated variability in the UV and optical lend support to this hypothesis. If this is the case, the estimated jet luminosity can increase by up to a factor of 8.

- For the standard cold accretion disc we have: $L_{d}=$ $1.7 \times 10^{-3}$. This estimate was obtained by Chaty et al. (2003) with a fit of the optical to EUV spectrum with a multicolour blackbody model. The inner disc temperature was found to be very low $k T \sim 20 \mathrm{eV}$, suggesting that either the inner disc is truncated at $\sim 350 R_{\mathrm{S}}$, or that the bulk of the accretion power is driven away in a coronal outflow and the disc is left very cold.

- Finally, for the luminosity of the X-ray emitting plasma, or corona, we have: $L_{\mathrm{x}}=1.2 \times 10^{-3}$, assuming isotropy.

Summing up all the three above spectral components, we find that the bolometric luminosity of the source is:

$L_{\mathrm{bol}}=L_{\mathrm{j}}+L_{\mathrm{d}}+L_{\mathrm{x}} \simeq 3 \times 10^{-3}$

The total mass accretion rate onto the black hole can be estimated from the observed luminosity of the cold disc component. We define the Eddington-scaled accretion rate $\dot{m}=\epsilon \dot{M} c^{2} / L_{\text {Edd }}$ where $\epsilon$ is the Newtonian accretion efficiency $\epsilon=1 / 12$. If the cold, geometrically thin and optically thick disc is indeed truncated at $R_{\text {in }}=350 R_{\mathrm{S}}$, as the spectral analysis of Chaty et al. (2003) suggests, then standard accretion formulae (Frank, King and Raine 2002) give:

$\dot{m}=4 \epsilon \frac{R_{\text {in }}}{R_{\mathrm{S}}} L_{d} \sim 0.2$.

On the other hand, if the accretion disc extends down to the innermost stable orbit $\left(R_{\mathrm{in}}=3 R_{\mathrm{S}}\right)$, but is sandwiched by a powerful corona, where a fraction $f_{\mathrm{H}}$ of the power is dissipated, then we can estimate that

$f_{\mathrm{H}}=\left(1+\frac{L_{d}(1-\eta)}{L_{\mathrm{x}}}\right)^{-1}$.

The $\eta$ parameter is only poorly constrained, but in any case $f_{\mathrm{H}}$ should be very large in order to explain the low inner disc temperature (Merloni, Di Matteo \& Fabian 2000). Taking $\eta \simeq 0.9$ as indicated by the time dependent model, we obtain $f_{\mathrm{H}}=0.88$, and, for the total mass accretion rate,

$\dot{m}=4 \epsilon \frac{R_{\mathrm{in}}}{R_{\mathrm{S}}} \frac{L_{d}}{\left(1-f_{\mathrm{H}}\right)} \sim 0.015$.

In both cases, the estimated accretion rate is much larger than the observed bolometric luminosity. Thus, most of 
the accretion power is not radiated and the source appears to be radiatively inefficient.

The important issue, however, would be to determine whether the missing accretion power escapes the system in the (low radiative efficiency) jet or in other forms of non-radiative losses, such as a slow wind, or large scale convective motions, or advection into the black hole. The answer to this question resides in the exact determination of the jet kinetic power. Unfortunately, there are major uncertainties in this determination, mainly because the jet radiative efficiency is not known. The jet is expected to be a poor radiator because most of the energy is lost in adiabatic expansion, thus, although the radiation from the jet represents a small fraction of the bolometric luminosity the jet could dominate the energetics. Both theory and observations indicate that the efficiency can be extremely low (as low as $\epsilon_{\mathrm{j}} \sim 10^{-4}$ see Celotti and Fabian 1991), but all present estimates are model dependent. In general they indicate radiative efficiencies of the order of $\epsilon_{\mathrm{j}} \sim 0.01$. For the case of XTE J1118+480 this would already imply that the total jet power dominates over the X-ray luminosity. Obviously, if the jet efficiency is lower, or the jet spectrum extends to shorter wavelength, or beaming effects are important, the jet dominance can easily be much larger. As discussed above, the analysis of our time dependent modeling strongly requires $f_{\mathrm{x}} \lesssim 0.1$, corresponding to a jet efficiency $\epsilon_{\mathrm{j}} \lesssim 3 \times 10^{-3}$.

In the case of the truncated disc plus inner hot inner flow model, the derived $\dot{m}$ sets an upper limit to the jet power, in the case where all the accretion power is lost in the jet: $P_{\mathrm{j}} / L_{\mathrm{x}} \sim \dot{m} / L_{\mathrm{x}} \sim 200$. On the other hand, if the jet power is comparatively modest e.g. $P_{\mathrm{j}} / L_{\mathrm{x}} \sim 10$ as assumed in the simulation of Fig. 1 this would imply that, although the jet dominates over the X-ray emission, it does not dominate as a power sink. If the dominant power sink is, for example, advection onto the black hole, the mass accretion rate we derive appears somewhat large to be consistent with ADAF models (Narayan \& Yi 1994). However, it is important to notice that the observed accretion rate is very sensitive to the value of the inner disc radius (see eq. 231), which is actually poorly constrained. Moreover, the critical accretion rate for ADAF models above which the ADAF solution breaks down scales as $\alpha^{2}$ (Rees et al. 1982; Narayan \& Yi 1995), and the unknown standard $\alpha$ parameter could be larger than usually considered leading to the existence of solutions at higher $\dot{m}$ than previously thought. Finally, we note that the dynamics of the ADAF solutions coupled with jets have not been worked out yet. The structure of the accretion flow as well as the (in)stability of the solution at high $\dot{m}$ are likely to be strongly affected by the presence of the jet. Although an advection-dominated accretion flow is in many aspects consistent with our analysis, it should certainly differ considerably from the standard solution.

In the case of the accretion disc plus patchy corona (or coronal outflow) model the $\dot{m}$ derived assuming $\eta=$ 0.9 is consistent with all of the non-radiated power driving the jet. However, when we attempted to fit the optical-UV spectrum of XTEJ1118+480 with the disc corona solution of Merloni \& Fabian (2002), we found that it was not possible to produce inner disc temperatures as low as observed $(\sim 20 \mathrm{eV})$. The coronal outflow model could be reconciled with the EUV spectral data only if an even larger fraction of the power was extracted from the disc into the corona/jet, requiring $f_{\mathrm{H}}$ to be very close to 1 . However, in the framework of magnetic coronae powered by MHD turbulent disc, having $f \sim 1$ also requires a very large (magnetic) viscosity (Merloni 2003), and implies an even stronger jet dominance of the total energy budget of the source.

An alternative way to obtain a low inner disc temperature together with reasonable value of $f_{\mathrm{H}}$ would be that the cold disc does not extend down to the last stable orbit. Instead, below a few tens of $R_{\mathrm{S}}$ the disc is truncated and the flow becomes very inefficient. However, such a picture would be very similar to the truncated disc plus hot inner flow scenario discussed above, except that the jet would be launched from the external accretion disc corona.

\section{SOME WIDER IMPLICATIONS OF THE MODEL}

\subsection{Comparison with other sources}

The behaviour of XTE J1118+480 demonstrates that the accretion flow onto a black hole can lead to a strong jet with little disk emission. A similar situation occurs in the massive elliptical galaxy M87 where powerful jets are associated with an otherwise weak galactic nucleus. The Bondi accretion rate onto the supermassive black hole in M87, deduced from the properties of the surrounding gas with Chandra observations (Di Matteo et al 2002), can give the observed jet power, deduced from the cavities made by them in the surrounding intracluster medium, provided that accretion efficiency is 0.1 or more. Assuming that the flow is in a steady state, such a high efficiency argues that most of the accreting gas flows to within a few gravitational radii of the black hole. Since thick hot flows are unstable to mass loss over a wide range af radii ( Begelman \& Blandford 1999; Quataert \& Gruzinov 2000; Stone, Pringle Begelman 2000), this favours a magnetically-dominated cold disk flow in the case of M87. By similarity it argues for a magnetically-dominated cold disc in XTE J1118+480 as well. The reservoir and presumably the base of the jet must also lie within a few gravitational radii of the black hole in both cases.

As mentioned in the Introduction, the low state of many Galactic Black Hole systems appears to contain a powerful jet. When systems drop below about one per cent of the Eddington accretion rate the disc may be magnetically dominated (Merloni \& Fabian 2002; Livio, Pringle \& King 2003), with a fast jet taking most of the gravitational energy released. This may also apply to massive black holes, as illustrated above with M87. The issue of how apparently well-fed massive black holes in galactic nuclei are feeble sources of electromagnetic radiation has been a puzzle for some years (Fabian \& Canizares 1988; Fabian \& Rees 1995; Di Matteo et al 2001, 2003; Loewenstein et al 2001; Pellegrini et al 2003). Most of these objects do however have jetted radio emission (Franceschini, Vercellone \& Fabian 1998) and a solution in which the accretion power is principally car- 
ried away by jets is a strong possibility. Unless the energy contained in black hole spin is a key factor, then magnetically-dominated discs should be part of that solution.

Further time-dependent studies of the multiwavelength behaviour in other stellar-mass and massive black hole systems may show similar behaviour to that of XTE $1118+480$. Some examples which already offer tantalizing behaviour are the Galactic microquasar GRS1915+105 (Mirabel et al., 1998; Klein-Wolt et al., 2002), the Galactic binary GX339-4 which has also displayed rapid optical flickering (Motch et al 1982) and the blazar 3C120 which shows X-ray dips before radio jet events are seen (Marscher et al 2002).

\subsection{Jet-dominated sources in the low/hard state}

Whatever the actual structure of the accretion flow, we have shown that during the outburst of XTE J1118+480, the total kinetic jet power should dominate over the X-ray luminosity, and could possibly be the dominant repository of the accretion power. There are additional independent arguments in favour of jet dominance in low/hard state sources and in XTE J1118+480 in particular. Based on the observed radio flux $\left(L_{\mathrm{R}}\right)$ and Xray correlation observed in hard states sources (Falcke \& Biermann 1996; Gallo, Fender \& Pooley, 2003), as well as on standard synchrotron formulae (Heinz \& Sunyaev, 2003), Fender, Gallo \& Jonker (2003) have shown that, provided that advection into the black hole horizon and/or convective motions do not store a large fraction of the accretion power, there should exist a critical accretion rate, $\dot{m}_{\mathrm{cr}}$, below which an accreting black hole is jet-dominated.

The exact value for the critical accretion rate could be inferred from the observations, if we knew the total jet power at a certain X-ray luminosity, and is given by $\dot{m}_{\mathrm{cr}}=2 P_{\mathrm{j}}^{2} / L_{\mathrm{x}}$, corresponding to a critical X-ray luminosity $L_{\mathrm{x}, \mathrm{cr}}=\dot{m}_{\mathrm{cr}} / 2$. Fender et al. (2001) derived a lower limit for the jet to X-ray power ratio in XTE J1118+480: $P_{j} / L_{x}=0.2$, and FGJ03 used this conservative estimates to determine the value of the critical rate $\dot{m}_{\text {cr }} \simeq 7 \times 10^{-5}$. However such a low value of the critical luminosity leads to several problems.

First, as shown in FGJ03, during the transition from a disc to a jet-dominated state, the dependence of the $\mathrm{X}$ ray luminosity on the accretion rate changes from being $L_{\mathrm{x}} \propto \dot{m}^{2}$, the right scaling for radiatively inefficient flows, to $L_{\mathrm{x}} \propto \dot{m}$, the scaling for radiatively efficient flows (see Fig. 1 of FGJ03). This would imply that with $L_{\mathrm{x}} \sim 10^{-3}$, XTE J1118+480 should be a radiatively efficient system. As discussed above, there is however strong observational evidence of the contrary.

Second, during the transition from jet to disc dominated state, the jet power changes from $P_{j} \propto \dot{m}$ which is the natural scaling expected in most jet models, to the seemingly unphysical $P_{j} \propto \dot{m}^{1 / 2}$.

Furthermore, black holes in the hard state should show some kind of spectral transition in the X-ray band at the critical luminosity $L_{\mathrm{x}, \mathrm{cr}} \sim 3 \times 10^{-5}$, due to the drastic changes in emission mechanisms that are needed to account for the different scalings of $L_{\mathrm{x}}$ with the accretion rate. The observations of low/hard state sources at such low luminosities are few and hard to perform, however no indication of any dramatic spectral change in any hard state source down to quiescent level has ever been reported (Kong et al., 2002; Hameury et al., 2003)

In fact, the only physical transition that we do actually observe is the transition between the hard and the soft state that occurs at luminosities of at least a few percent of Eddington luminosity (Maccarone 2003). We believe that, if the above mentioned difficulties are to be solved, then $\dot{m}_{\mathrm{cr}}$ has to correspond to luminosities that are comparable to, or larger than, hard-to-soft state transition luminosities.

For the case of XTE J1118+480, instead of using the lower limit for the jet to X-ray power $\left(P_{j} / L_{x}=0.2\right)$, we can adopt the much larger value $P_{j} / L_{x} \sim 10$ required by our variability model. Then we find $\dot{m}_{\mathrm{cr}} \sim 0.2$, involving a transition at $L_{\mathrm{x}, \mathrm{cr}}=\dot{m}_{\mathrm{cr}} / 2 \sim 0.1$. This is in agreement with the idea that the transition from jet dominated to X-ray dominated states occurs at luminosities similar or slightly higher than the hard to soft state transition. We stress that the arguments of FGJ03 holds only as long as the $L_{\mathrm{R}}-L_{\mathrm{x}}$ correlation is valid i.e. not in the soft-state. There is thus no reason to associate the disc dominated sate of FGJ03 to the soft state.

If, as we suggest, the critical luminosity $L_{\mathrm{x}, \mathrm{cr}}$ is at or above the hard to soft state transition luminosity, then the disc dominated state of FGJ03 does not exist. Moreover, although one can speculate that the transition to soft state may be be triggered by the loss of the jet dominance, there is a priori no reason for the critical luminosity $L_{\mathrm{x}, \mathrm{cr}}$ to be coincident with the hard to soft state transition luminosity. In other words, the observed presence of a hard-to-soft state transition does not provide an upper limit to the jet power, but only a lower limit.

We also note that the above discussion was made neglecting any contribution from advection and other possible non-radiative losses. However, in jet-dominated sources the jet power scales proportionally to the accretion rate, as is the case for any adiabatic loss. Therefore the effects of advection (and/or convection) would not change the scalings of jet and X-ray powers provided that we replace the accretion rate $\dot{m}$ with $\dot{m}_{\text {eff }}=\dot{m}\left(1-f_{\text {ad }}\right)$, where $f_{\text {ad }}$ is the fraction of accretion power that is advected into the black hole. In particular, the critical X-ray luminosity $L_{\mathrm{x}, \mathrm{cr}}$, although corresponding to a larger 'real' $\dot{m}$, would remain unchanged.

To conclude, the central requirement of our model i.e. that in XTE J1118+480 the jet power dominates over the X-ray luminosity, appears to be supported by the observations of hard state black holes. Then, if the arguments of FGJ03 are correct, an important consequence of the jet dominance in XTE J1118+480 is that all hard state sources are jet-dominated (in the sense that the jet power dominates over the X-ray power). This jet dominance also implies that all hard state sources should be radiatively inefficient.

It is not clear whether most of the accretion energy is channeled into the jet or across the black hole event horizon. The $L_{\mathrm{x}} \propto \dot{m}_{\text {eff }}^{2}$ dependence of the X-ray luminosity 
(also observed in low-luminosity AGN, as inferred from the multivariate radio-X-rays-mass correlation, Merloni, Heinz \& Di Matteo 2003; Falcke, Körding \& Markoff 2003) is similar to what is predicted by radiatively inefficient accretion model. The reason for this inefficiency could be advection into the jet as well as advection into the black hole.

\section{CONCLUSIONS}

We have shown that the puzzling optical/X-ray correlations of XTE J1118+480, can be understood in terms of a common energy reservoir for both the jet and the Comptonizing electrons. For illustration purpose, we have presented a specific shot noise variability model that reproduces all the main observed features of the multiwavelength correlated variability.

Our time dependent model is fairly general, and our main conclusions hold regardless of the specific geometrical and dynamical properties of the system. The main results can be summarized as follows:

- Any energy reservoir model for XTE J1118+480 requires that the total jet power dominates over the X-ray luminosity. In particular, assuming that the compact jet synchrotron emission extends up to the optical band, this implies a radiative efficiency for the jet $\epsilon_{\mathrm{j}} \lesssim 3 \times 10^{-3}$. Following the same line of arguments as FGJ03, we showed that this situation is likely and probably represents a common feature of all black holes in the low-hard state

- The range of typical variability timescales of dissipation rate of the X-ray emitting plasma is consistent with the dynamical times of an accretion disc between $\sim 10$ and 100 Schwarzschild radii (for a $10 M_{\odot}$ black hole). As expected, the X-ray variability can be associated with either a hot, thick inner accretion flow or with a patchy corona.

- As jet launching requires large-scale coherent magnetic structures, the energy dissipation in the jet should vary on longer time scales. Indeed, the derived range of timescales associated with the dissipation in the jet extends to timescales more than 10 times larger than the $\mathrm{X}$-ray emitting one.

- By combining the information obtained from our time variability model with (time averaged) spectral analysis we conclude that, whatever the accretion geometry, the whole disc-corona-jet system must be radiatively inefficient. It is therefore possible, in principle, that the system is energetically dominated by the jet, as suggested by FGJ03, although whether the bulk of the accretion power is advected into the black hole or into the jet remains at present an open question.

- In terms of specific dynamical models, we conclude that the observed properties of XTE J1118+480 during its low/hard state outburst are consistent with either an inner hot, quasi spherical, radiatively inefficient flow, from which the jet originates (Meier 2001), surrounded by a geometrically-thin, optically-thick cold disc, or with a powerful patchy, outflowing corona on top of an extremely cold standard thin disc. In the first case, multicolour disc fits to the UV/EUV spectrum indicate a very large inner disc radius, implying a large total accretion rate $(\dot{m} \simeq 0.2$ ), which might be in conflict with the hypothesis of standard advection dominated flow theory. In the second case, in order to reproduce the very low inner disc temperature, an (uncomfortably) extreme coronal power is needed, together with substantial relativistic bulk motion of the coronal plasma, both possibly associated with very high magnetic viscosity.

\section{ACKNOWLEDGEMENTS}

JM acknowledges financial support from PPARC. We are grateful to Annalisa Celotti and Jim Pringle for useful discussions.

\section{REFERENCES}

Balbus S. A., Hawley J. F., 1998, RvMP, 70, 1

Blandford R. D., Begelman M. C., 1999, MNRAS, 303, L1

Blandford R. D., Znajek R. L., 1977, MNRAS, 179, 433

Blandford R.D., Königl A., 1979, ApJ, 232, 34

Blandford R. D., Payne D. G., 1982, MNRAS, 199, 883

Celotti A., Fabian A. C., 1993, MNRAS, 264, 228

Chaty S., Haswell C.A., Malzac J., Hynes R.I., Shrader

C.R., Cui W., 2003, MNRAS, 346, 689.

Corbel S., Fender R.P., Tzioumis A.K., Nowak M., McIntyre V., Durouchoux P., Sood R., 2000, A\&A, 359, 251

Corbel S., Nowak M. A., Fender R. P., Tzioumis A. K., Markoff S., 2003, A\&A, 400, 1007

Di Matteo T., Blackman E.G., Fabian A.C., 1997, MNRAS, 291, L23

Di Matteo T., Johnstone R. M., Allen S. W., Fabian A. C., 2001, ApJ, 550, L19

Di Matteo T., Allen S. W., Fabian A. C., Wilson A. S., Young A. J., 2003, ApJ, 582, 133

Esin A. A., McClintock J. E., Drake J. J., Garcia M. R., Haswell C. A., Hynes R. I., Muno M. P., 2001, ApJ, 555,483

Fabian A. C., Canizares C. R., 1988, Natur, 333, 829

Fabian A. C., Rees M. J., 1995, MNRAS, 277, L55

Fender R. et al., 1999, ApJ, 519, L165

Fender R. P., Hjellming R. M., Tilanus R. P. J., Pooley G. G., Deane J. R., Ogley R. N., Spencer R. E., 2001, MNRAS, 322, L23

Fender R. P., Gallo E., Jonker P. G., 2003, MNRAS, 343, L99

Fender, R., 2004, to appear in 'Compact Stellar X-Ray Sources', eds. W.H.G. Lewin and M. van der Klis, Cambridge University Press, astro-ph/0303339

Franceschini A., Vercellone S., Fabian A. C., 1998, MNRAS, 297, 817

Falcke H., Körding E., Markoff S., A\&A, in press, astro-ph/0305335

Frank J., King A., Raine D. J., 2002, Accretion Power in Astrophysics, 3rd ed., Cambridge University Press, Cambridge.

Gallo E., Fender R. P., Pooley G. G., 2003, MNRAS, 344,60 
Georganopoulos M., Aharonian F. A., Kirk J. G., 2002, A\&A, 388, L25

Falcke H., Biermann P. L., 1996, A\&A, 308, 321

Gierlinski M., Zdziarski A. A., Done C., Johnson W. N., Ebisawa K., Ueda Y., Haardt F., Phlips B. F., 1997, MNRAS, 288, 958

Hameury J.-M., Barret D., Lasota J.-P., McClintock J. E., Menou K., Motch C., Olive J.-F., Webb N., 2003, A\&A, 399, 631

Hjellming R.M., Johnston K.J., 1988, ApJ, 328, 600

Hynes R. I. et al., 2003, MNRAS, 345, 292

Kanbach G., Straubmeier C., Spruit H. C., Belloni T., 2001, Nature, 414, 180

Kong A. K. H., McClintock J. E., Garcia M. R., Murray S. S., Barret D., 2002, ApJ, 570, 277

King A.R., Pringle J.E., West R.G., Livio M., 2003, MNRAS, in press, astro-ph/0311035

Klein-Wolt M., Fender R. P., Pooley G. G., Belloni T., Migliari S., Morgan E. H., van der Klis M., 2002, MNRAS, 331, 745

Loewenstein M., Mushotzky R. F., Angelini L., Arnaud K. A., Quataert E., 2001, ApJ, 555, L21

Livio M., Ogilvie G. I., Pringle J. E., 1999, ApJ, 512, 100

Livio M., Pringle J. E., King A. R., 2003, ApJ, 593, 184

Maccarone T. J., Coppi P. S., 2002, MNRAS, 336, 817

Maccarone T. J., 2003, A\&A, 409, 697

Malzac J., Beloborodov A. M., Poutanen J., 2001, MNRAS, 326, 417

Malzac J., Belloni T., Spruit H. C., Kanbach G., 2003, A\&A, 407, 335

Markoff S., Falcke H., Fender R., 2001, A\&A, 372, L25

Marscher A. P., Jorstad S. G., McHardy I. M., Aller M. F., Balonek T. J., Sokolov A. S., 2002, AAS, 34, 1246

McClintock J. E., Garcia M. R., Caldwell N., Falco E. E., Garnavich P. M., Zhao P., 2001a, ApJ, 551, L147

McClintock J. E. et al., 2001b, ApJ, 555, 477

McClintock, J.E., Remillard, R.A., 2004, to appear in 'Compact Stellar X-ray Sources', eds. W.H.G. Lewin and M. van der, astro-ph/0306213

Meier D. L., 2001, ApJ, 548, L9

Merloni A., Di Matteo T., Fabian A.C., 2000, MNRAS,318, L15

Merloni A., Fabian A. C., 2001, MNRAS, 321, 549

Merloni A., Fabian A. C., 2002, MNRAS, 332, 165

Merloni A., 2003, MNRAS, 341, 1051

Merloni A., Heinz S., di Matteo T., 2003, MNRAS, 345, 1057

Mirabel I. F., Dhawan V., Chaty S., Rodriguez L. F., Marti J., Robinson C. R., Swank J., Geballe T., 1998, A\&A, 330, L9

Motch C., Ilovaisky S. A., Chevalier C., 1982, A\&A, 109, L1

Narayan R., Yi I., 1994, ApJ, 428, L13

Narayan R., Yi I., 1995, ApJ, 452, 710

Negoro H., Kitamoto S., Takeuchi M., Mineshige S., 1995, ApJ, 452, L49

Pellegrini S., Baldi A., Fabbiano G., Kim D.-W., 2003, ApJ, 597, 175

Poutanen, J., 1998, in Abramowicz M.A., Björnsson G.,
Pringle J., eds, Theory of Black hole Accretion Disk. Cambridge Univ. Press, Cambridge, p. 100

Poutanen J., Fabian A.C., 1999, MNRAS, 306, L31

Quataert E., 1998, ApJ, 500, 978

Quataert E., Gruzinov A., 1999, ApJ, 520, 248

Quataert E., Gruzinov A., 2000, ApJ, 539, 809

Rees, M. J., Phynney, E. S., Begelman, M. C., Blandford, R. D., 1982, Nature, 295, 17

Remillard R., Morgan E., Smith D., Smith, E., 2000, IAU Circ., 7389, 2

Shapiro S. L., Lightman A. P., Eardley D. M., 1976, ApJ, 204, 187

Spruit H. C., Kanbach G., 2002, A\&A, 391, 225

Stirling A.M., Spencer R.E., de la Force C.J., Garrett M.A., Fender R.P., Ogley R.N., 2001, MNRAS, 327, 1273.

Stone J. M., Pringle J. E., Begelman M. C., 1999, MNRAS, 310, 1002

Tananbaum H., Gursky H., Kellogg E., Giacconi R., Jones C., 1972, ApJ, 177, L5

Tanaka Y., Lewin W.H.G., 1995, in 'X-ray Binaries', eds. W.H.G. Lewin, J. van Paradjis, E. van den Heuvel. Cambridge University Press.

Uttley P., McHardy I. M., 2001, MNRAS, 323, L26 